\title{
A Discrete Dynamical Model of Signed Partitions
}

\author{
G. Chiaselotti, G. Marino, P. A. Oliverio, and D. Petrassi \\ Dipartimento di Matematica, Università della Calabria, Via Pietro Bucci, Cubo 30B, 87036 Arcavacata di Rende, Italy \\ Correspondence should be addressed to G. Marino; gmarino@unical.it
}

Received 28 November 2012; Accepted 19 February 2013

Academic Editor: Luigi Muglia

Copyright (C) 2013 G. Chiaselotti et al. This is an open access article distributed under the Creative Commons Attribution License, which permits unrestricted use, distribution, and reproduction in any medium, provided the original work is properly cited.

We use a discrete dynamical model with three evolution rules in order to analyze the structure of a partially ordered set of signed integer partitions whose main properties are actually not known. This model is related to the study of some extremal combinatorial sum problems.

\section{Introduction}

A dynamical system is by definition a system whose state changes with time $t$. We have a discrete dynamical system when $t$ is an integer or a natural number, and the elements of the system can be obtained in the form $u_{t+1}=F\left(u_{t}\right)$, where $F$ is some global function which describes the evolution rule of the system (see [1]). In a series of very recent works, the theory of discrete dynamical systems has been applied in several contexts. In [2], the theory of discrete dynamical systems is applied in order to analyze some models of concurrent computing systems. In [3], a dynamical model of parallel computation on bi-infinite time scale with an approach similar to two-sided symbolic dynamics is constructed. In [4], the authors analyze the orbit structure of parallel discrete dynamical systems over directed dependency graphs, with Boolean functions as global functions. In [5], the authors extend the manner of defining the evolution update of discrete dynamical systems on Boolean functions, without limiting the local functions to being dependent restrictions of a global one. Finally, in [6] is given a complete characterization of the orbit structure of parallel discrete dynamical systems with maxterm and minterm Boolean functions as global functions. In [7], the authors have introduced the poset $\left(S(n, d, r)\right.$, ) of all the signed integer partitions $r \geq a_{r} \geq \cdots \geq$ $a_{1} \geq 0 \geq b_{1} \geq \cdots \geq b_{n-r} \geq-(n-r)$, where the positive parts and the negative parts are all distinct between them and the number of nonzero parts is exactly $d$. In this case, the partial order $\sqsubseteq$ is that on the components. The concept of signed integer partition has been introduced in [8] and studied in [9] from an arithmetical point of view. In [7, 10], it is shown that the structure of the lattice $S(n, d, r)$ is strictly related to the study of some combinatorial extremal sum problems. In this paper, we study some particular type of signed partitions (specifically, of the signed partitions of $S(n, d, r)$ ) by adopting the interesting point of view of the discrete dynamical models. In particular, we analyze the lattice $S(n, d, r)$ as a discrete dynamical model with three local evolution rules whose dynamics is studied in a sequential mode. The way to study a lattice of classical partitions as a discrete dynamic model having some particular evolution rules is implicit in [11], where Brylawski proposed a dynamical approach to study the lattice $L_{B}(n)$ of all the partitions of the fixed positive integer $n$ with the dominance ordering. In such a context, a configuration of the system is represented by an ordered partition of an integer $n$, that is, a decreasing sequence $a=$ $\left(a_{1}, \ldots, a_{n}\right)$ having sum $n$, whose blocks of the corresponding Young diagram are considered as mobile sand grain, and the movement of a sand grain, respects the following rules.

Rule 1 (vertical rule). One grain can move from a column to the next column if the difference of height of these two columns is greater than or equal to 2 .

Rule 2 (horizontal rule). If a column containing $p+1$ grains is followed by a sequence of columns containing $p$ grains and then one column containing $p-1$ grains, then one grain of the first column can slip to the last column.

In this paper, we prove that the covering relation in the lattice $S(n, d, r)$ is uniquely determined by three evolution rules of our discrete dynamical model. We articulate the proof 
of this result by contradiction by using only the first-order logic. This method of proof is very laborious; however, its advantage is that it can be easily implemented on a proof assistant checker based on the first-order logic (e.g., Mizar). The paper is articulated as follows. In Section 2, we recall some basic definitions and preliminary results, for example, the definition of $S(n, d, r)$ and some of its properties. In Section 3, we explain how to see the signed partitions of $S(n, d, r)$ as configurations of our discrete dynamical model and we also describe its evolution rules. In Section 4 , we assume by contradiction that there is a generic element $w^{\prime}$ of $S(n, d, r)$ that covers another generic element $w \in S(n, d, r)$ but $w^{\prime}$ is not generated by $w$ with none of our evolution rules. From this assumption we deduce several propositions and conditions that we use in order to prove our principal result, that is, Theorem 4.

\section{Definitions and Preliminary Results}

If $(X, \leq)$ is a poset and $x, y \in X$, we write $y>x$ (or $x<$ $y$ ) if $y$ covers $x$. Now we briefly recall the definition of the lattice $S(n, r)$ that we have introduced in [7] in a more formal context. Let $n$ and $r$ be two nonnegative integers such that $r \leq n$.

We call $(n, r)$-string a $n$-tuplet of integers

$$
a_{r} \cdots a_{1} \mid b_{1} \cdots b_{n-r}
$$

such that

(i) $a_{1}, \ldots, a_{r} \in\{1, \ldots, r, 0\}$;

(ii) $b_{1}, \ldots, b_{n-r} \in\{-1, \ldots,-(n-r), 0\}$;

(iii) $a_{r} \geq \cdots \geq a_{1} \geq 0 \geq b_{1} \geq \cdots \geq b_{n-r}$;

(iv) the unique element in (1) which can be repeated is 0 .

If $w$ is a $(n, r)$-string, we call parts of $w$ the integers $a_{r}, \ldots, a_{1}, b_{1} \cdots b_{n-r}$, nonnegative parts of $w$ the integers $a_{r}, \ldots, a_{1}$, and nonpositive parts of $w$ the integers $b_{1} \cdots b_{n-r}$. We set $w_{+}=a_{r} \cdots a_{1} \mid$ and $w_{-}=\mid b_{1} \cdots b_{n-r}$. In some cases, we do not distinguish between nonnegative and nonpositive parts of $w$, and we write more simply $w=l_{1} \cdots l_{n}$ instead of $w=a_{r} \cdots a_{1} \mid b_{1} \cdots b_{n-r}$. We also denote by $|w|_{>}$the number of parts of $w$ that are strictly positive, with $|w|_{<}$the number of parts of $w$ that are strictly negative, and we set $\|w\|=$ $|w|_{>}+|w|_{<} . S(n, r)$ is the set of all the $(n, r)$-strings. If $w=$ $a_{r} \cdots a_{1} \mid b_{1} \cdots b_{n-r}$ and $w^{\prime}=a_{r}^{\prime} \cdots a_{1}^{\prime} \mid b_{1}^{\prime} \cdots b_{n-r}^{\prime}$ are two $(n, r)-$ strings, we set $w_{+}=w_{+}^{\prime}$ if $a_{i}^{\prime}=a_{i}$ for all $i=r, \ldots, 1, w_{-}=w_{-}^{\prime}$ if $b_{j}^{\prime}=b_{j}$ for all $j=1, \ldots, n-r$, and $w=w^{\prime}$ if $w_{+}=w_{+}^{\prime}$ and $w_{-}=w_{-}^{\prime}$. On $S(n, r)$, we consider the partial order on the components that we denote by $\sqsubseteq$. To simplify the notations, in all the numerical examples the integers on the right of the vertical bar | will be written without minus sign. Since $(S(n, r)$, ᄃ) is a finite distributive lattice, it is also graded, with minimal element $0 \cdots 0 \mid 12 \cdots(n-r)$ and maximal element $r(r-1) \cdots 21 \mid 0 \cdots 0$.

We recall now the concept of involution poset (see $[12,13]$ for some recent studies on such class of posets). An involution poset (IP) is a poset $(X, \leq, c)$ with a unary operation $c: x \in$ $X \mapsto x^{c} \in X$, such that
(I1) $\left(x^{c}\right)^{c}=x$, for all $x \in X$;

(I2) if $x, y \in X$ and if $x \leq y$, then $y^{c} \leq x^{c}$.

The map $c$ is called complementation of $X$ and $x^{c}$ the complement of $x$. Let us observe that if $X$ is an involution poset, by (I1) it follows that $c$ is bijective and by (I1) and (I2) it holds that if $x, y \in X$ are such that $x<y$, then $y^{c}<x^{c}$. If $(X, \leq, c)$ is an involution poset and if $Z \subseteq X$, we will set $Z^{c}=\left\{z^{c}: z \in Z\right\}$. We note that if $X$ is an involution poset, then $X$ is a self-dual poset because from (I1) and (I2) it follows that if $x, y \in X$, we have that $x \leq y$, if and only if $y^{c} \leq$ $x^{c}$, and this is equivalent to say that the complementation is an isomorphism between $X$ and its dual poset $X^{*}$. In [7], it has been shown that $(S(n, r)$, ᄃ) is an involution poset and its complementation map $c$ is the following:

$$
\begin{aligned}
& \left(a_{k} \cdots a_{1} 0 \cdots 0 \mid 0 \cdots 0 b_{1} \cdots b_{l}\right)^{c} \\
& \quad=a_{r-k}^{\prime} \cdots a_{1}^{\prime} 0 \cdots 0 \mid 0 \cdots 0 b_{1}^{\prime} \cdots b_{n-r-l}^{\prime}
\end{aligned}
$$

where $\left\{a_{1}^{\prime}, \ldots, a_{r-k}^{\prime}\right\}$ is the usual complement of $\left\{a_{1}, \ldots, a_{k}\right\}$ in $\{1, \ldots, r\}$, and $\left\{b_{1}^{\prime}, \ldots, b_{n-r-l}^{\prime}\right\}$ is the usual complement of $\left\{b_{1}, \ldots, b_{l}\right\}$ in $\{-1, \ldots,-(n-r)\}$ (e.g., in $S(7,4)$, we have that $\left.(4310 \mid 001)^{c}=2000 \mid 023\right)$. If $d$ is an integer such that $0 \leq$ $d \leq n$, we set now $S(n, d, r)=\{w \in S(n, r):\|w\|=d\}$. Its easy to see that $(S(n, d, r)$, $)$ is a sublattice of $(S(n, r), \sqsubseteq)$ and obviously $|S(n, d, r)|=\left(\begin{array}{l}n \\ d\end{array}\right)$.

We call signed Young diagrams (briefly SYD) an ordered couple $D=D_{1}: D_{2}$, where $D_{1}$ is a Young diagram of an integer partition built with decreasing columns instead with decreasing rows, and $D_{2}$ is a Young diagram of an integer partition built with increasing columns. In the sequel, we will call pile a column of a Young diagram and grain a square of a pile. For example,

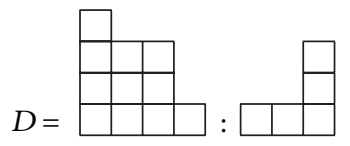

is determined by the partitions 4331 and 113. If $w=$ $a_{r} \cdots a_{1} \mid b_{1} \cdots b_{n-r} \in S(n, r)$, we denote by $D(w)$ the signed Young diagram $D(w)=D^{+}(w): D^{-}(w)$, where $D^{+}(w)$ is the Young diagram of the partition $\left(a_{r}, \ldots, a_{1}\right)$ and $D^{-}(w)$ is the Young diagram of the partition $\left(-b_{1}, \ldots,-b_{n-r}\right)$. Now, if $D=D_{1}: D_{2}$ is a SYD, where $\left(a_{t}, \ldots, a_{1}\right)$ is the decreasing partition having $D_{1}$ as Young diagram and $\left(\left(-b_{1}\right), \ldots,\left(-b_{s}\right)\right)$ is the increasing partition having $D_{2}$ as Young diagram, we set $p(D)=a_{t} \cdots a_{1} \mid\left(-b_{1}\right) \cdots\left(-b_{s}\right)$ and we call $p(D)$ the signed partition of $D$. We say that a signed Young diagram $D$ is a $(n, r)$-SYD if it results that $p(D) \in S(n, r)$ and we denote with $Y S(n, r)$ the set of all the $(n, r)-S Y D$. It is clear that the map $\phi$ : $w \mapsto D(w)$ is a bijection between $S(n, r)$ and $Y S(n, r)$, whose inverse function is the map $\psi: D \mapsto p(D)$; therefore, in the sequel, we will identify the elements of $S(n, r)$ with the $(n, r)$ SYDs of $Y S(n, r)$. If $A$ and $B$ are two usual Young diagrams, we set $A \subseteq B$ if $A$ is a subdiagram of $B$. If $D=D_{1}: D_{2}$ and $D^{\prime}=D_{1}^{\prime}: D_{2}^{\prime}$ are two SYDs, we set

$$
D \sqsubseteq D^{\prime} \Longleftrightarrow D_{1} \subseteq D_{1}^{\prime}, \quad D_{2} \supseteq D_{2}^{\prime} .
$$


Therefore, it is clear that we can identify $(S(n, r)$, ᄃ) with $(Y S(n, r)$, ㄷ). Now we set $Y S(n, d, r)=\{D(w): w \in S(n, d, r)\}$. Obviously we can identify $(Y S(n, d, r)$, ) with $(S(n, d, r)$, ). If $(H)$ is a statement, in the sequel we denote by $\neg(H)$ the negation of $(H)$.

\section{Description of the Evolution Rules}

In our dynamic model, the set of all configurations is exactly $Y S(n, d, r)$. Our goal is to define some rules of evolution that starting from the minimum of $Y S(n, d, r)$ allow us to reconstruct the Hasse diagram of $Y S(n, d, r)$ (and therefore to determine the covering relations in $S(n, d, r))$.

Let $w=a_{r} \cdots a_{1} \mid b_{1} \cdots b_{n-r} \in S(n, d, r)$ and $D=D(w)=$ $D^{+}(w): D^{-}(w)$. If $1 \leq i \leq r$, we call $i$ th-plus pile of $D$, and we denote it by $C_{+}(i, D)$, the ith pile of $D^{+}(w)$ that corresponds to part $a_{i}$ of $w$ and, if $1 \leq j \leq n-r$, we call $j$ th-minus pile of $D$, and we denote it by $C_{-}(j, D)$, the $j$ th pile of $D^{-}(w)$ that corresponds to part $b_{j}$ of $w$. We call the pile $C^{+}(i, D)$ plus singleton pile if $a_{i}=1$ and $C^{-}(j, D)$ minus singleton pile if $b_{j}=1$. Let us note that if there exists a plus singleton pile, then it is necessarily unique, analogously for a minus singleton pile. If $1<i \leq r$, we set $\Delta_{i}^{+}(w)=a_{i}-a_{i-1}$ and we call $\Delta_{i}^{+}(w)$ the plus height difference of $w$ in $i$. If $1<j \leq n-r$, we set $\Delta_{j}^{-}(w)=\left|b_{j}\right|-\left|b_{j-1}\right|$ and we call $\Delta_{j}^{-}(w)$ the minus height difference of $w$ in $j$. If $1<i \leq r$, we say that $w$ has a plus cliff at $i$ if $\Delta_{i}^{+}(w) \geq 2$. If $1<j \leq n-r$, we say that $w$ has a minus cliff at $j$ if $\Delta_{j}^{-}(w) \geq 2$.

Remark 1. When we apply the following rules to one element $w \in S(n, r)$, we impose that there is an "invisible" extra pile in the imaginary place $(r+1)$ of $D^{+}(w)$ having exactly $r+1$ grains. This is only a formal trick for decreas the number of rules necessary for our model; therefore, the $(r+1)$ th pile must be not considered as a part of $w$.

\subsection{Evolution Rules.}

Rule 1. If the $i$ th-plus pile has at least one grain and if $w$ has a plus cliff at $i+1$, then one grain must be added on the $i$ th-plus pile:

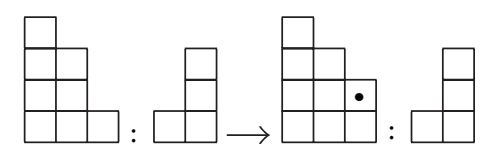

Rule 2. If there is not a plus singleton pile and there is a minus singleton pile, then the latter must be shifted to the side of the lowest non empty plus pile:

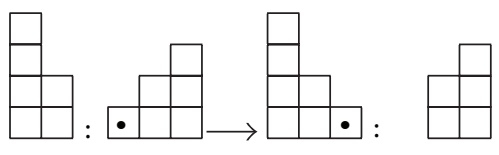

Rule 3. One grain must be deleted from the $j$ th-minus pile if $w$ has a minus cliff at $j$ :

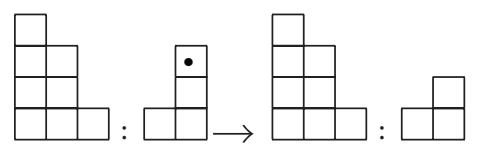

Remark 2. (i) Under the hypothesis in Rule 3, the jth plus must have at least 2 grains.

(ii) In Rule 2, the lowest non empty plus pile can also be the invisible column in the place $r+1$. In this case, all the plus piles are empty and a possible minus singleton pile must be shifted in the place $r$.

\section{Main Result}

In the sequel, we write $w \rightarrow{ }^{k} w^{\prime}\left(\right.$ or $w^{\prime}=w \rightarrow{ }^{k}$ ) to denote that $w^{\prime}$ is an $n$-tuplet of integers obtained from $w$ applying the Rule $k$, for $k=1,2,3$, and that $\neg\left(w \rightarrow{ }^{k} w^{\prime}\right)$ if the condition $w \rightarrow{ }^{k} w^{\prime}$ is false. We also set

$$
\nabla(w)=\left\{w^{\prime}: w \longrightarrow^{k} w^{\prime}, k=1,2,3\right\} .
$$

Proposition 3. If $w \in S(n, d, r)$, then $\nabla(w) \subseteq\left\{w^{\prime} \in S(n, d, r)\right.$ : $\left.w^{\prime}>w\right\}$.

Proof. Let $w=a_{r} \cdots a_{1} \mid b_{1} \cdots b_{n-r} \in S(n, d, r), a_{r+1}=r+1$ (the invisible pile in the place $r+1)$, and $D=D(w)$. We distinguish the three possible cases related to the previous rules.

Case 1. Let us assume that $r \geq i \geq 1, a_{i} \neq 0$, and that $w$ has a plus cliff at $i+1$. If $w^{\prime}=w \rightarrow{ }^{1}$, then $w^{\prime}=a_{r} \cdots a_{i+1}\left(a_{i}+\right.$ 1) $a_{i-1} \cdots a_{1} \mid b_{1} \cdots b_{n-r}$. It is clear that $\left\|w^{\prime}\right\|=d$ because $a_{i} \neq 0$. Since there is a plus cliff at $i+1$, we have $a_{i+1}-a_{i} \geq 2$; hence, $a_{i+1} \geq a_{i}+2>a_{i}+1>a_{i}>a_{i-1}$, and this implies that $w^{\prime} \in$ $S(n, d, r)$. We must show now that $w^{\prime}$ covers $w$ in $S(n, d, r)$. Since $w$ and $w^{\prime}$ differ between them only in the place $i$ for $a_{i}$ and $a_{i}+1$, respectively, it is clear that there does not exist an element $z \in S(n, d, r)$ such that $w \sqsubset z \sqsubset w^{\prime}$. Hence, $w^{\prime}>w$.

Case 2. Let us assume that in $D(w)$ there is not a plus singleton pile and that there is a minus singleton pile (we say $C^{-}(j, D)$, for some $1 \leq j \leq n-r$ ). Since $a_{r+1}=r+1$, we can assume that $a_{i+1}>0, a_{i}=$ 0 , for some $1 \leq i \leq r$. This means that $w$ has the following form: $w=a_{r} \cdots a_{i+1} 00 \cdots 0 \mid 0 \cdots 0(-1) b_{j+1} \cdots b_{n-r}$, where $a_{i+1}>1$ (otherwise $D(w)$ has a plus singleton pile). Applying Rule 2 to $w$, we obtain $w^{\prime}=w \rightarrow^{2}$, where $w^{\prime}=a_{r} \cdots a_{i+1} 10 \cdots 0 \mid 0 \cdots 00 b_{j+1} \cdots b_{n-r}$. It is clear then that $w^{\prime} \in S(n, r)$ and $\left\|w^{\prime}\right\|=d$ since $w^{\prime}$ is obtained from $w$ with only a shift of the pile- 1 to the left in the place $i$. Let us note that the only elements $z_{1}, z_{2} \in S(n, r)$ such that $w \sqsubset z_{1} \sqsubset w^{\prime}$ and $w \sqsubset z_{2} \sqsubset w^{\prime}$ are $z_{1}=a_{r} \cdots a_{i+1} 10 \cdots 0 \mid 0 \cdots 0(-1) b_{j+1} \cdots b_{n-r}$ and $z_{2}=a_{r} \cdots a_{i+1} 00 \cdots 0 \mid 0 \cdots 00 b_{j+1} \cdots b_{n-r}$, but $\left\|z_{1}\right\|=d+1$ and $\left\|z_{2}\right\|=d-1$; hence, $z_{1}, z_{2}$ are not elements of $S(n, d, r)$. This implies that $w^{\prime}$ covers $w$ in $S(n, d, r)$.

Case 3. If $1<j \leq n-r$ and $w$ has a minus cliff at $j$, we apply Rule 3 to $w$ on the pile $C^{-}(j, D)$ and we obtain $w^{\prime}=w \rightarrow^{3}$, 
where $w^{\prime}=a_{r} \cdots a_{1} \mid b_{1} \cdots b_{j-1}\left(b_{j}+1\right) b_{j+1} \cdots b_{n-r}$. Since $w$ has a minus cliff at $j$, we have $-b_{j}+b_{j-1}=\left|b_{j}\right|-\left|b_{j-1}\right| \geq 2$; therefore, $w^{\prime} \in S(n, r)$ because $0 \geq b_{j-1} \geq b_{j}+2>b_{j}+1>b_{j}>b_{j+1}$ and $\left\|w^{\prime}\right\|=d$ since $b_{j} \leq-2$ implies $b_{j}+1<0$. As in Case 1 , we note that $w^{\prime}$ covers $w$ in $S(n, d, r)$ because they differ between them only for a grain in the place $j$.

The main result of this paper is to prove that if $w, w^{\prime} \in$ $S(n, d, r)$, then $w^{\prime}$ covers $w$ in $S(n, d, r)$ if and only if $w \rightarrow{ }^{k} w^{\prime}$ for some $k \in\{1,2,3\}$. Obviously this result can be reformulated as follows.

Theorem 4. If $w \in S(n, d, r)$, then $\nabla(w)=\left\{w^{\prime} \in S(n, d, r)\right.$ : $\left.w^{\prime}>w\right\}$.

By Proposition 3, to prove Theorem 4, it suffices to show that $\left\{w^{\prime} \in S(n, d, r): w^{\prime}>w\right\} \subseteq \nabla(w)$. To show this inclusion, we proceed by contradiction as follows. We start with the following hypotheses:

$$
\begin{gathered}
w, w^{\prime} \in S(n, d, r), \quad w=a_{r} \cdots a_{1} \mid b_{1} \cdots b_{n-r}, \\
w^{\prime}=a_{r}^{\prime} \cdots a_{1}^{\prime} \mid b_{1}^{\prime} \cdots b_{n-r}^{\prime}, \quad w^{\prime}>w
\end{gathered}
$$

and we also assume that

$$
\neg\left(w \longrightarrow^{k} w^{\prime}\right) \quad \text { for each } k=1,2,3 .
$$

Then the proof of Theorem 4 will consist into show that the hypotheses (9) and the conditions (10) always lead to contradictory cases, so that it must be necessarily verified the inclusion $\left\{w^{\prime} \in S(n, d, r): w^{\prime}>w\right\} \subseteq \nabla(w)$.

We will articulate the proof of Theorem 4 in several cases. Each possible case will be placed in the form of a proposition and we will obtain a contradiction in all cases, showing, hence, the thesis of the theorem. Before proceeding, let us note that in some cases we also use the alternative notations $w=l_{1} \cdots l_{n}$ and $w^{\prime}=l_{1}^{\prime} \cdots l_{n}^{\prime}$.

We set now

$$
\begin{gathered}
\Omega_{1}(w)=\left\{i \in\{1, \ldots, r\}: a_{i}>0, a_{i+1}-a_{i} \geq 2\right\}, \\
\Omega_{3}(w)=\left\{j \in\{2, \ldots, n-r\}: b_{j-1}-b_{j} \geq 2\right\}, \\
\Lambda^{+}\left(w, w^{\prime}\right)=\left\{h \in\{1, \ldots, r\}: a_{h}^{\prime}>a_{h}\right\}, \\
\Lambda^{-}\left(w, w^{\prime}\right)=\left\{l \in\{1, \ldots, n-r\}: b_{l}^{\prime}>b_{l}\right\} .
\end{gathered}
$$

Formalization of Rules 1, 2, and 3.

The description of Rules 1, 2, and 3 in terms of parts of $w$ and $w^{\prime}$ is the following:

(1) $w \rightarrow{ }^{1} w^{\prime}$ is equivalent to

$\Omega_{1}(w) \neq \emptyset \operatorname{AND}\left(\exists i \in \Omega_{1}(w):\left(a_{i}^{\prime}=a_{i}+1\right)\right.$ AND $\left.\left(\Lambda^{+}\left(w, w^{\prime}\right) \backslash\{i\}=\Lambda^{-}\left(w, w^{\prime}\right)=\emptyset\right)\right) ;$

(2) $w \rightarrow{ }^{2} w^{\prime}$ is equivalent to

(for all $k \in\{1, \ldots, r\} a_{k} \neq 1$ ) AND

$\left(\exists j \in\{1, \ldots, n-r\}\right.$ such that $\left.b_{j}=-1\right)$ AND $\left(\exists i \in\{1, \ldots, r\}\right.$ such that $\left.a_{i+1}>0, a_{i}=0\right)$ AND

$\left(\Lambda^{+}\left(w, w^{\prime}\right) \backslash\{i\}=\Lambda^{-}\left(w, w^{\prime}\right) \backslash\{j\}=\emptyset, a_{i}^{\prime}=1, b_{j}^{\prime}=0\right) ;$

(3) $w \rightarrow{ }^{3} w^{\prime}$ is equivalent to

$\Omega_{3}(w) \neq \emptyset$ AND $\left(\exists j \in \Omega_{3}(w):\left(b_{j}^{\prime}=b_{j}+1\right)\right.$ AND $\left.\left(\Lambda^{+}\left(w, w^{\prime}\right)=\Lambda^{-}\left(w, w^{\prime}\right) \backslash\{j\}=\emptyset\right)\right)$.

Negation of Rules 1, 2, and 3.

(1) $\neg\left(w \rightarrow{ }^{1} w^{\prime}\right) \Leftrightarrow\left[\left(1 \mathrm{~A}^{\prime}\right)\right.$ OR (1B) $]$,

where

$(1 \mathrm{~B})$ is $\left(\Omega_{1}(w)=\emptyset\right)$,

$\left(1 \mathrm{~A}^{\prime}\right)$ is $\left[\Omega_{1}(w) \neq \emptyset \mathrm{AND}\right.$ (for all $i \in \Omega_{1}(w)\left(a_{i}^{\prime} \neq a_{i}+\right.$ 1) $\left.\left.\mathrm{OR}\left(\Lambda^{+}\left(w, w^{\prime}\right) \backslash\{i\} \neq \emptyset \mathrm{OR} \Lambda^{-}\left(w, w^{\prime}\right) \neq \emptyset\right)\right)\right]$.

(2) $\neg\left(w \rightarrow{ }^{2} w^{\prime}\right) \Leftrightarrow\left[\left(2 \mathrm{~A}^{\prime}\right)\right.$ OR (2B) OR (2C) OR (2D)], where

$\left(2 \mathrm{~A}^{\prime}\right)$ is (for all $k \in\{1, \ldots, r\} a_{k} \neq 1$ ) AND

$\left(\exists j \in\{1, \ldots, n-r\}\right.$ such that $\left.b_{j}=-1\right)$ AND

$\left(\exists i \in\{1, \ldots, r\}\right.$ such that $\left.a_{i+1}>0, a_{i}=0\right)$ AND

$\left(\left(\Lambda^{+}\left(w, w^{\prime}\right) \backslash\{i\} \neq \emptyset\right.\right.$ OR $\left.\Lambda^{-}\left(w, w^{\prime}\right) \backslash\{j\} \neq \emptyset\right)$ OR $a_{i}^{\prime} \neq 1$ OR $\left.b_{j}^{\prime} \neq 0\right)$;

(2B) is $\left(\exists k \in\{1, \ldots, r\}\right.$ such that $\left.a_{k}=1\right)$;

(2C) is (for all $j \in\{1, \ldots, n-r\} b_{j} \neq-1$ );

(2D) is (for all $i \in\{1, \ldots, r\} a_{i+1}=0$ OR $a_{i}>0$ ).

(3) $\neg\left(w \rightarrow{ }^{3} w^{\prime}\right) \Leftrightarrow\left[\left(3 \mathrm{~A}^{\prime}\right)\right.$ OR (3B) $]$,

where

$(3 \mathrm{~B})$ is $\left(\Omega_{3}(w)=\emptyset\right)$;

$\left(3 \mathrm{~A}^{\prime}\right)$ is $\left[\Omega_{3}(w) \neq \emptyset \mathrm{AND}\right.$ (for all $j \in \Omega_{3}(w)\left(b_{j}^{\prime} \neq b_{j}+\right.$ 1) $\left.\left.\mathrm{OR}\left(\Lambda^{+}\left(w, w^{\prime}\right) \neq \emptyset \mathrm{OR} \Lambda^{-}\left(w, w^{\prime}\right) \backslash\{j\} \neq \emptyset\right)\right)\right]$.

we set

$$
\begin{gathered}
(1.1 \mathrm{~b}): w=00 \cdots 0 \mid b_{1} \cdots b_{n-r}, \\
(1.2 \mathrm{~b}): w=r(r-1) \cdots(k+1) k \cdots 0 \mid b_{1} \cdots b_{n-r} \\
\quad \text { for some } k \in\{1, \ldots, r\} .
\end{gathered}
$$

Proposition 5. Consider (1B) $\Rightarrow((1.1 b)$ OR (1.2b)).

Proof. The condition $\Omega_{1}(w)=\emptyset$ means that for all $i \in$ $\{1, \ldots, r\}, a_{i}=0$ or $0 \leq a_{i+1}-a_{i} \leq 1$ (i.e., $a_{i+1}-a_{i}=0$ or $\left.a_{i+1}-a_{i}=1\right)$. We have then the following possibilities:

(j) $a_{i}=0$;

(jj) $a_{i+1}-a_{i}=0$ (and therefore $a_{i+1}=a_{i}=0$ since $w \epsilon$ $S(n, r))$;

(jjj) $a_{i+1}=a_{i}+1$

If $a_{i}=0$ for all $i \in\{1, \ldots, r\}$ then (12) holds. Let us assume therefore that $k \in\{1, \ldots, r\}$ is the smallest index such that $a_{k}>0$; hence, $a_{r}>a_{r-1}>\cdots>a_{k+1}>a_{k}>0$ and $a_{k-1}=$ $\cdots=a_{1}=0$. This means that for all indexes $i \in\{r, r-1, \ldots, k+$ $1, k\}$ holds necessarily (jjj). In particular, for $i=r$ we have $r+1=a_{r+1}=a_{r}+1$; hence, $a_{r}=r$. Now, if $i=r-1$, $r=a_{r}=a_{r-1}+1$, that is, $a_{r-1}=r-1$. Iterating (13) follows. 
We set

$$
(2.1 \mathrm{~d}): w=r(r-1) \cdots 21 \mid b_{1} \cdots b_{n-r} .
$$

Proposition 6. Consider (2D) $\Leftrightarrow(2.1 d)$.

Proof. By (2D), if we take $i=r$, we have $a_{r+1}=0$ or $a_{r}>0$; therefore, it is necessarily $a_{r}>0$ because by hypothesis, $a_{r+1}=$ $r+1>0$. Thus, still by (2D), for $i=r-1$, we have $a_{(r-1)+1}=$ $a_{r}=0$ or $a_{r-1}>0$; therefore, it must be $a_{r-1}>0$. By iterating, we can deduce that $a_{r}>0, a_{r-1}>0, \ldots, a_{1}>0$; hence, $a_{1}=1$, $a_{2}=2, \ldots, a_{r}=r$ because $w$ is an element of $S(n, r)$, that is, exactly (14). On the other side, if $w=r(r-1) \cdots 2 \quad 1 \mid b_{1} \cdots b_{n-r}$, then it is immediate to note that $(2 \mathrm{D})$ is verified.

We set

$$
\begin{gathered}
(3.1 \mathrm{~b}): w=a_{r} \cdots a_{1} \mid 00 \cdots 0, \\
(3.2 \mathrm{~b}): w=a_{r} \cdots a_{1} \mid 00 \cdots 0(-1)(-2) \cdots(-l) \\
\quad \text { for some } l \in\{1, \ldots, n-r\} .
\end{gathered}
$$

Proposition 7. Consider (3B) $\Rightarrow((3.1 b)$ OR (3.2b)).

Proof. The condition $\Omega_{3}(w)=\emptyset$ means that for all $j \in$ $\{2, \ldots, n-r\}, 0 \leq b_{j-1}-b_{j} \leq 1$ (i.e., $b_{j-1}-b_{j}=0$ or $b_{j-1}-b_{i}=1$ ). We have then the following possibilities:

(i) $b_{j-1}-b_{j}=0$ (and therefore $b_{j-1}=b_{i}=0$ since $w \epsilon$ $S(n, r))$

(ii) $b_{j-1}=b_{j}+1$.

If (i) holds for all $j \in\{2, \ldots, n-r\}$, then (15) holds. We can assume therefore that $k \in\{2, \ldots, n-r\}$ is the smallest index such that (ii) holds. Let us observe then that if in a place $j \in$ $\{2, \ldots, n-r\}$, (ii) holds; then also in the places $j+1, \ldots, n-r$, (ii) holds. In fact, if $b_{j}=b_{j-1}-1$ and by absurd $b_{j}=b_{j+1}=$ 0 , then $b_{j-1}=1$ which is a contradiction because $0 \geq b_{j-1}$. Therefore, if (ii) holds in $j$, then it also holds in $j+1$, and therefore, by iteration, it holds for $j+1, j+2, \ldots, n-r$. Now we assume at first that $k=2$. In this case, (ii) holds for all indexes in $\{2, \ldots, n-r\}$; therefore, $b_{1}=0$ or $b_{1}=-1$ and $b_{2}=b_{1}-1$, $b_{3}=b_{2}-1 \cdots$. If $b_{1}=0$, then $w=a_{r} \cdots a_{1} \mid 0(-1)(-2) \cdots(-(n-$ $r)+1)$ and if $b_{1}=-1$, then $w=a_{r} \cdots a_{1} \mid(-1)(-2) \cdots(-(n-r))$; thus in both cases (ii) holds. Let now $k \geq 3$. Then $b_{k-1}=b_{k-2}=$ 0 (because in the place $k-1$ (i) holds) and hence $0=b_{k-1}=$ $b_{k-2}=\cdots=b_{2}=b_{1}$. Moreover, since (i) holds in the places $k, k+1, \ldots, n-r$, we have $b_{k}=b_{k-1}-1=0-1=-1$; therefore, $b_{k+1}=b_{k}-1=-2, \ldots, b_{n-r}=b_{n-r-1}-1=-(n-r-k)-1=-l$, where $l=n-r-k+1$. This completes the proof.

Proposition 8. Consider $(3.1 b) \Rightarrow\left(w_{-}=w_{-}^{\prime}=\mid 00 \cdots 0\right)$.

Proof. Since $w \sqsubset w^{\prime}$, it must be $0 \geq b_{j}^{\prime} \geq b_{j}$, where $b_{j}=0$ for all $j \in\{1, \ldots, n-r\}$ because (15) implies $w=a_{r} \cdots a_{1} \mid 00 \cdots 0$. Therefore, $b_{j}=b_{j}^{\prime}=0$ for all $j \in\{1, \ldots, n-r\}$; that is, $w^{\prime}=$ $a_{r}^{\prime} \cdots a_{1}^{\prime} \mid 00 \cdots 0$.

\section{Proposition 9. Consider}

(i) (1.1b) AND (2B) $\Rightarrow$ absurd,

(ii) (1.1b) AND (2.1d) $\Rightarrow$ absurd, (iii) (2C) AND (3.2b) $\Rightarrow$ absurd,

(iv) (1.1b) AND (3.1b) $\Rightarrow$ absurd,

(v) (1.2b) AND (3.1b) $\Rightarrow$ absurd.

Proof. (i), (ii), and (iii) are obvious.

(iv) By (12) and (15), it follows that $w=0 \cdots 0 \mid 0 \cdots 0$, absurd.

(v) By Proposition 8, we have $w_{-}^{\prime}=w_{-}$. Then, since $w \sqsubset w^{\prime},\left\|w^{\prime}\right\|=\|w\|$ and $w_{-}^{\prime}=w_{-}$, by (13) we deduce that $w_{+}^{\prime}=w_{+}$. Hence, the absurd $w^{\prime}=w$.

Proposition 10. Consider (1.2b) AND (2B) $\Rightarrow$ (2.1d).

Proof. By (2B), it follows that in (13) it must be $k=1$; therefore, $w=r(r-1) \cdots 21 \mid b_{1} \cdots b_{n-r}$.

Proposition 11. Consider (2.1d) AND (3.2b) $\Rightarrow$ absurd.

Proof. By (14), it is immediate to observe that $w_{+}^{\prime}=w_{+}$ because $w \sqsubset w^{\prime}$. Then by (16), since $w_{+}^{\prime}=w_{+},\left\|w_{-}^{\prime}\right\|=\left\|w_{-}\right\|$ and $w \sqsubset w^{\prime}$, it must also be $w_{-}^{\prime}=\mid 00 \cdots 0(-1)(-2) \cdots(-l)$. Hence, we obtain $w=w^{\prime}$, absurd.

Proposition 12. Consider $\left(2 A^{\prime}\right) \Rightarrow$ absurd.

Proof. We set

(F) [(for all $\left.k \in\{1, \ldots, r\} a_{k} \neq 1\right)$ AND $(\exists j \in\{1, \ldots, n-$ $r\}$ such that $\left.b_{j}=-1\right)$ AND $(\exists i \in\{1, \ldots, r\}$ such that $\left.\left.a_{i+1}>0, a_{i}=0\right)\right]$

(G) $\left(\Lambda^{+}\left(w, w^{\prime}\right) \backslash\{i\} \neq \emptyset\right.$ OR $\left.\Lambda^{-}\left(w, w^{\prime}\right) \backslash\{j\} \neq \emptyset\right)$;

(H) $\left(a_{i}^{\prime} \neq 1\right.$ OR $\left.b_{j}^{\prime} \neq 0\right)$.

Then $\left(2 \mathrm{~A}^{\prime}\right)$ is equivalent to ((F) AND $\left.(\mathrm{G})\right)$ OR ((F) AND (H)). We show at first that $((\mathrm{F})$ AND $(\mathrm{H}))$ leads to a contradiction. Next we show that $((\mathrm{F})$ AND $(\mathrm{G})$ AND $\neg(\mathrm{H}))$ also leads to an absurd. This will prove the thesis;

$((\mathrm{F})$ AND $(\mathrm{H})) \Rightarrow$ absurd.

By (F), it follows that $w$ satisfies the hypothesis of Rule 2; therefore, by Proposition 3 we can take an element $z \in$ $S(n, d, r)$ such that $z=w \rightarrow^{2}$. As in the proof of the Case 2 in Proposition 3, it results that $w$ and $z$ must have the following form:

$$
\begin{aligned}
& w=a_{r} \cdots a_{i+1} 00 \cdots 0 \mid 0 \cdots 0(-1) b_{j+1} \cdots b_{n-r}, \\
& z=a_{r} \cdots a_{i+1} 10 \cdots 0 \mid 0 \cdots 00 b_{j+1} \cdots b_{n-r},
\end{aligned}
$$

where $a_{i+1}>1$ and $b_{j+1}<-1$. We distinguish now several cases.

(I) $b_{j}^{\prime} \neq 0$. Then $b_{j}^{\prime} \leq-1$, and also $b_{j}^{\prime} \geq b_{j}=-1$ because $w \sqsubset w^{\prime}$; hence $b_{j}^{\prime}=-1$.

We note now that

$$
a_{r}^{\prime}>0, \ldots, a_{i+1}^{\prime}>0
$$


because $a_{r}^{\prime} \geq a_{r}>0, \ldots, a_{i+1}^{\prime} \geq a_{i+1}>0$. Moreover, since $b_{j}^{\prime}=-1$, it follows that

$$
-1=b_{j}^{\prime}>b_{j+1}^{\prime}>\cdots>b_{n-r}^{\prime}
$$

because $w^{\prime} \in S(n, r)$. Now, since $w \in S(n, d, r)$, from the form of $w$ it results that $d=(r-i)+(n-r-j+1)$. Moreover, since also $w^{\prime} \in S(n, d, r)$, we have $\left\|w^{\prime}\right\|=d=(r-i)+(n-r-j+1)$. Therefore, since in (19), there are exactly $r-i$ inequalities and in (20) there are exactly $n-r-j+1$ inequalities; it follows that

$$
w^{\prime}=a_{r}^{\prime} \cdots a_{i+1}^{\prime} 00 \cdots 0 \mid 0 \cdots 0(-1) b_{j+1}^{\prime} \cdots b_{n-r}^{\prime} .
$$

(II) $a_{i}^{\prime}=0$. Since $w \sqsubset w^{\prime}$, by (17) we have two possible cases: $w^{\prime}$ has the form (21) or

$$
w^{\prime}=a_{r}^{\prime} \cdots a_{i+1}^{\prime} 00 \cdots 0 \mid 0 \cdots 00 b_{j+1}^{\prime} \cdots b_{n-r}^{\prime}
$$

with $a_{i+1}^{\prime} \geq a_{i+1}>1$ and $0 \geq b_{j+1}^{\prime}$. If (22) holds, then $\left\|w^{\prime}\right\| \leq$ $(r-i)+(n-r-j)=d-1$, which is absurd because $w^{\prime} \in$ $S(n, d, r)$; hence it must hold necessarily (21).

(III) $a_{i}^{\prime}>1$. Since $w \sqsubset w^{\prime}$ and $d=\|w\|=\left\|w^{\prime}\right\|$, by (17) it follows that

$$
w^{\prime}=a_{r}^{\prime} \cdots a_{i+1}^{\prime} a_{i}^{\prime} 0 \cdots 0 \mid 0 \cdots 00 b_{j+1}^{\prime} \cdots b_{n-r}^{\prime} .
$$

Then from the hypothesis $a_{i}^{\prime} \geq 2$ and by (18), we have $w \sqsubset$ $z \sqsubset w^{\prime}$, which is a contradiction.

Hence the unique possibility is that $w^{\prime}$ has the form (21). Since $w^{\prime}>w$, we have $a_{k}^{\prime} \geq a_{k}$ for $k=r, \ldots, i+2, i+1$ and $b_{l}^{\prime} \geq b_{l}$ for $l=j+1, j+2, \ldots, n-r$. Moreover, at least one inequality of the type $a_{k}^{\prime}>a_{k}$ or the type $b_{l}^{\prime}>b_{l}$ must hold. We distinguish now the several cases.

$(\Delta 1)$ We assume that a unique inequality of the type $a_{p}^{\prime}>$ $a_{p}$ holds for some $p=r, \ldots, i+2, i+1$. Therefore, we have $a_{k}^{\prime}=a_{k}$ for all $k \neq p$ and $b_{l}^{\prime}=b_{l}$ for all $l=j+1, j+2, \ldots, n-r$. Then, since $a_{p+1}=a_{p+1}^{\prime}>a_{p}^{\prime}>a_{p}>0$ (let us note that the previous inequalities also hold if $p=r$ because $a_{r+1}=r+1=$ $\left.a_{r+1}^{\prime}\right)$, we have that $a_{p+1}-a_{p} \geq 2$ and $a_{p}>0$. Hence $w$ satisfies the hypotheses of Rule 1 in the place $p$ and so $w \rightarrow{ }^{1} u$, where

$$
\begin{aligned}
u=a_{r} \cdots a_{p+1}\left(a_{p}+1\right) a_{p-1} \cdots a_{i+1} 0 \cdots 0 \mid & \\
0 & \cdots 0(-1) b_{j+1} \cdots b_{n-r} \\
(= & a_{r}^{\prime} \cdots a_{p+1}^{\prime}\left(a_{p}+1\right) a_{p-1}^{\prime} \cdots a_{i+1}^{\prime} 0 \cdots 0 \\
& \left.0 \cdots 0(-1) b_{j+1}^{\prime} \cdots b_{n-r}^{\prime}\right),
\end{aligned}
$$

with $u \in S(n, d, r)$. Now, if $a_{p}^{\prime}=a_{p}+1$, it results that $w^{\prime}=u$; therefore, $w \rightarrow^{1} w^{\prime}$, which is a contradiction with the hypothesis $\neg\left(w \rightarrow{ }^{1} w^{\prime}\right)$. Otherwise, if $a_{p}+1<a_{p}^{\prime}$, then we have $w \sqsubset u \sqsubset w^{\prime}$, which is absurd because $w^{\prime}>w$.

$(\Delta 2)$ If a unique inequality of the type $b_{q}^{\prime}>b_{q}$ holds for some $q=j+1, j+2, \ldots, n-r$, we deduce a contradiction as in the proof of the previous case by using Rule 3 instead of Rule 1.

$(\Delta 3)$ We assume now that at least two strict inequalities among the $a_{k}^{\prime} \geq a_{k}$ and $b_{l}^{\prime} \geq b_{l}$ hold, and let us also assume that the first (by left) of such strict inequalities is $a_{p}^{\prime}>a_{p}$ for some $p \in\{r, \ldots, i+1\}$. We consider then the following $(n, r)$ string:

$$
\begin{gathered}
v=a_{r} \cdots a_{p+1}\left(a_{p}+1\right) a_{p-1} \cdots a_{i+1} 0 \cdots 0 \\
0 \cdots 0(-1) b_{j+1} \cdots b_{n-r} .
\end{gathered}
$$

Since $a_{p}^{\prime}>a_{p}$ is the first strict inequality by left, we have $a_{p+1}^{\prime}=a_{p+1}$ (also if $p=r$ ); hence $a_{p+1}=a_{p+1}^{\prime}>a_{p}^{\prime} \geq$ $a_{p}+1>a_{p}>a_{p-1}$ because $a_{p}>0$; this implies that $v \in S(n, r)$; therefore $v \in S(n, d, r)$ because $\|v\|=\|w\|=d$. Moreover, it is clear that $w \sqsubset v$. Let us observe now that $v \sqsubset w^{\prime}$; in fact, by hypothesis, $w^{\prime}$ must have at least another place $q \neq p$ where $a_{q}^{\prime}>a_{q}$ or $b_{q}^{\prime}>b_{q}$ and $v$ differs by $w$ only in the place $p$. This shows that $w \sqsubset v \sqsubset w^{\prime}$, with $v \in S(n, d, r)$, which is absurd because $w^{\prime}>w$.

$(\Delta 4)$ If at least two strict inequalities among the $a_{k}^{\prime} \geq a_{k}$ and $b_{l}^{\prime} \geq b_{l}$ hold and the first (by left) of such strict inequalities is $b_{p}^{\prime}>b_{p}$ for some $p \in\{j+1, j+2, \ldots, n-r\}$, then we deduce a contradiction in the same way as in the previous case.

This concludes the proof that ((F) AND $(\mathrm{H})) \Rightarrow$ absurd. To complete the proof we show now that ((F) AND (G) AND $\neg(\mathrm{H})) \Rightarrow$ absurd.

Since $(\mathrm{H})$ is false, we have $a_{i}^{\prime}=1$ and $b_{j}^{\prime}=0$; therefore, $w$ has the form (17) and $w^{\prime}=a_{r}^{\prime} \cdots a_{i+1}^{\prime} 10 \cdots 0 \mid 0 \cdots$ $00 b_{j+1}^{\prime} \cdots b_{n-r}^{\prime}$. We take $z \in S(n, d, r)$ as in (18), so that $w$ and $z$ differ between them only in the places $i, j$ and $w \sqsubset z$. Moreover, since $w^{\prime}=a_{r}^{\prime} \cdots a_{i+1}^{\prime} 10 \cdots 0 \mid 0 \cdots 00 b_{j+1}^{\prime} \cdots b_{n-r}^{\prime}$, by $(\mathrm{G}),(18)$, and (17) it follows that $z \sqsubset w^{\prime}$. Therefore, we obtain $w \sqsubset z \sqsubset w^{\prime}$ that is in contrast with the hypothesis $w^{\prime}>w$.

We set now

$$
\begin{aligned}
& (1 \mathrm{~A}): \Omega_{1}(w) \neq \emptyset, \quad \forall i \in \Omega_{1}(w) \quad a_{i}^{\prime} \neq a_{i}+1, \\
& (3 \mathrm{~A}): \Omega_{3}(w) \neq \emptyset, \quad \forall j \in \Omega_{1}(w) \quad b_{j}^{\prime} \neq b_{j}+1 .
\end{aligned}
$$

Proposition 13. Consider
(i) $\left(1 A^{\prime}\right) \Leftrightarrow(1 A)$,
(ii) $\left(3 A^{\prime}\right) \Leftrightarrow(3 A)$.

Proof. (i) The implication $\Leftarrow$ is obvious. We assume now that $\left(1 \mathrm{~A}^{\prime}\right)$ holds and there exists $i \in \Omega_{1}(w)$ such that $a_{i}^{\prime}=a_{i}+1$ and also that $\Lambda^{+}\left(w, w^{\prime}\right) \backslash\{i\} \neq \emptyset$ (i.e., there exists $h \neq i$ such that $\left.a_{h}^{\prime}>a_{h}\right)$ or $\Lambda^{-}\left(w, w^{\prime}\right) \neq \emptyset$ (i.e., there exists $l$ such that $\left.b_{l}^{\prime}>b_{l}\right)$. Since $i \in \Omega_{1}(w)$, we can apply Rule 1 to $w$ and we can take $z=w \rightarrow{ }^{1}$. By Proposition 3, we know that $z \in S(n, d, r)$ and $z>w$. Moreover, since $a_{h}^{\prime}>a_{h}(h \neq i)$ or $b_{l}^{\prime}>b_{l}$, we also have $z \sqsubset w^{\prime}$; therefore, $w \sqsubset z \sqsubset w^{\prime}$, which is in contrast with the hypothesis $w^{\prime}>w$. Hence this proves that the condition 
$a_{i}^{\prime} \neq a_{i}+1$ must be verified for all $i \in \Omega_{1}(w)$, which is exactly (26).

(ii) The proof is similar to (i) applying Rule 3 to $w$ instead of Rule 1.

We set

$$
\begin{aligned}
& \text { (1a): } \Omega_{1}(w) \neq \emptyset, \quad \forall i \in \Omega_{1}(w) a_{i}^{\prime}=a_{i}, \\
& \text { (3a): } \Omega_{3}(w) \neq \emptyset, \quad \forall j \in \Omega_{3}(w) b_{j}^{\prime}=b_{j} .
\end{aligned}
$$

\section{Proposition 14. Consider}

(i) $(1 A) \Rightarrow(1 a)$

(ii) $(3 A) \Rightarrow(3 a)$.

Proof. (i) By (1A), we have $\Omega_{1}(w) \neq \emptyset$, and by the definition of $\Omega_{1}(w)$ this is equivalent to say that $w$ satisfies the hypotheses of Rule 1 for some $i \in\{1, \ldots, r\}$. Therefore, by Proposition 3 , there exists an element $z_{i} \in S(n, d, r)$ such that $w \rightarrow{ }^{1} z_{i}$, where $z_{i}=a_{r} \cdots a_{i+1}\left(a_{i}+1\right) a_{i-1} \cdots a_{1} \mid b_{1} \cdots b_{n-r}$ and obviously $w \sqsubset z_{i}$. Since $a_{i}^{\prime} \neq a_{i}+1$, we distinguish two cases.

(a) $a_{i}^{\prime}>a_{i}+1$. In this case $w \sqsubset z_{i} \sqsubset w^{\prime}$, that is in contrast with the hypothesis $w^{\prime}>w$.

(b) $a_{i}^{\prime}<a_{i}+1$. In this case $a_{i}^{\prime} \leq a_{i}$; hence $a_{i}^{\prime}=a_{i}$ because $a_{i}^{\prime} \geq a_{i}$ from the hypothesis $w^{\prime}>w$.

(ii) The proof of this case is similar to (i).

Lemma 15. Assume that (1a) holds. If $q \in\{2,3, \ldots, r, r+1\}$, $a_{q}^{\prime}=a_{q}$, and $a_{q-1}>0$, then $a_{q-1}^{\prime}=a_{q-1}$.

Proof. If $q-1 \in \Omega_{1}(w)$, then the thesis holds by the definition of (1a) in (28). We assume now that $q-1 \notin \Omega_{1}(w)$. Then $a_{q-1}=0$ or $0 \leq a_{(q-1)+1}-a_{q-1}=a_{q}-a_{q-1} \leq 1$, and since $a_{q-1}>0$, we necessarily have $0 \leq a_{q}-a_{q-1} \leq 1$. Now, if $a_{q}-a_{q-1}=0$, we have $a_{q}=a_{q-1}=0$ since $w \in S(n, r)$, which is in contradiction with $a_{q-1}>0$. Hence, it must be $a_{q}-a_{q-1}=1$; that is, $a_{q}=a_{q-1}+1$. Moreover, $a_{q}^{\prime}>a_{q-1}^{\prime}$ because $a_{q-1}^{\prime} \geq a_{q-1}>0$ and $w^{\prime} \in S(n, r)$. Therefore, since $a_{q}^{\prime}=a_{q}$, we have $a_{q-1}+1=a_{q}=a_{q}^{\prime}>a_{q-1}^{\prime}$, which implies $a_{q-1}+1>a_{q-1}^{\prime} \geq a_{q-1}$ because $w \sqsubset w^{\prime}$. By subtracting $a_{q-1}$ from each term of the previous inequalities, we obtain $1>$ $a_{q-1}^{\prime}-a_{q-1} \geq 0$; therefore, it must be $a_{q-1}^{\prime}=a_{q-1}$.

We set

$$
\begin{aligned}
& \text { (1.1a): } \exists l \in\{r, \ldots, 2\} \quad \text { such that } \\
& a_{l}>0 \text {, } \\
& w=a_{r} a_{r-1} \cdots a_{l+1} a_{l} 0 \cdots 0 \mid b_{1} \cdots b_{n-r}, \\
& w^{\prime}=a_{r} a_{r-1} \cdots a_{l+1} a_{l} a_{l-1}^{\prime} \cdots a_{1}^{\prime} \mid b_{1}^{\prime} \cdots b_{n-r}^{\prime} .
\end{aligned}
$$

Proposition 16. Consider (1a) $\Rightarrow$ (1.1a).

Proof. By (28), there exists at least one element $i \in \Omega_{1}(w)$. In this place we have $a_{i}>0$ and moreover $a_{i+1}-a_{i} \geq 2$. Since $w \in S(n, r)$, it must be $a_{1}=0$ or $a_{1}=1$. If $a_{1}=1$, then $a_{2}=$ $2, \ldots, a_{r}=r$, which is in contrast with $a_{i+1}-a_{i} \geq 2$. Hence $a_{1}=0$ and therefore there exists $l \in 2, \ldots, r$ such that $a_{l}>0$ and $a_{l-1}=\cdots=a_{1}=0$. We take $q=r+1$; that is, $q-1=r$. Since $a_{q}^{\prime}=a_{q}=r+1$ and $a_{q-1}=a_{r} \geq a_{l}>0$, by Lemma 15 it follows that $a_{q-1}^{\prime}=a_{q-1}$; that is, $a_{r}^{\prime}=a_{r}$. Let now $q=r$. If $r=l$, then we have the thesis otherwise $q=r>l$; that is, $q-1 \geq l$; therefore $a_{q-1} \geq a_{l}>0$ because $w \in S(n, r)$. Then since $a_{q}^{\prime}=$ $a_{r}^{\prime}=a_{r}=a_{q}$, we can apply Lemma 15 and we deduce $a_{q-1}^{\prime}=$ $a_{q-1}$; that is, $a_{r-1}^{\prime}=a_{r-1}$. By iteration the thesis follows.

Lemma 17. Assume that ( $3 a)$ holds. If $q \in\{2,3, \ldots, n-r-1\}$, $b_{q}^{\prime}=b_{q}<0$, then $b_{q+1}^{\prime}=b_{q+1}$.

Proof. If $q+1 \in \Omega_{3}(w)$, then the thesis holds by definition of (3a) in (29). We assume now that $q+1 \notin \Omega_{3}(w)$. Now, if $b_{q}-b_{q+1}=0$, we have $b_{q}=b_{q+1}=0$ since $w \in S(n, r)$, which is in contradiction with the hypothesis $b_{q}<0$. Hence it must be $b_{q}-b_{q+1}=1$; that is, $0>b_{q}=b_{q+1}+1$; therefore $0>b_{q+1}+1=$ $b_{q}=b_{q}^{\prime}>b_{q+1}^{\prime} \geq b_{q+1}$ since $w^{\prime} \in S(n, r)$ and $w \sqsubset w^{\prime}$. The last inequalities provide $b_{q+1}+1>b_{q+1}^{\prime} \geq b_{q+1}$. By subtracting $b_{q+1}$ from each term of the previous inequalities, we obtain $1>b_{q+1}^{\prime}-b_{q+1} \geq 0$; therefore it must be $b_{q+1}^{\prime}=b_{q+1}$.

Proposition 18. If ( $3 a$ ) holds, then $w$ has the following form: $w=a_{r} \cdots a_{1} \mid 0 \cdots 0 b_{l} b_{l+1} \cdots b_{n-r}$, with $b_{l}<0$ and $l \geq 2$. Moreover, if $b_{l} \leq-2$, we also have $w_{-}^{\prime}=w_{-}$.

Proof. By (3a) in (29), we know that $\Omega_{3}(w) \neq \emptyset$. Moreover, if $j \in \Omega_{3}(w)$, then $j \geq 2$ and $b_{j}<0$ because $b_{j-1}-b_{j} \geq 2$. Therefore, we can take the minimum $l \in 2, \ldots, n-r$ such that $b_{l}<0$; hence $b_{l-1}=\cdots=b_{1}=0$ and this means that $w=a_{r} \cdots a_{1} \mid 0 \cdots 0 b_{l} b_{l+1} \cdots b_{n-r}$. Assume now that $b_{l} \leq-2$. Since $b_{l-1}=0$, we have $b_{l-1}-b_{l} \leq 2$; therefore $l \in \Omega_{3}(w)$. By (3a), follows then that $b_{l}^{\prime}=b_{l}<0$. Now, if $l=n-r$, the thesis is true, otherwise, if $l \in\{2, \ldots, n-r-1\}$, by Lemma 17 we have $b_{l+1}^{\prime}=b_{l+1}<0$. Again, if $l+1=n-r$, the thesis is true; otherwise, if $l+1 \in\{2, \ldots, n-r-1\}$, by Lemma 17 we have $b_{l+2}^{\prime}=b_{l+2}<0$. Proceeding repeatedly in this way we obtain $b_{l}^{\prime}=b_{l}, b_{l+1}^{\prime}=b_{l+1}, \ldots, b_{n-r}^{\prime}=b_{n-r}$. Finally, since $b_{1}=\cdots=$ $b_{l-1}=0$ and $w \sqsubset w^{\prime}$, we also have $b_{1}^{\prime}=\cdots=b_{l-1}^{\prime}=0$. This proves that $w_{-}^{\prime}=w_{-}$.

Proposition 19. Consider ((2C) AND (3a)) $\Rightarrow w_{-}^{\prime}=w_{-}$.

Proof. (2C) implies that $w$ has the form $w=a_{r} \cdots a_{1} \mid 0 \cdots$ $0 b_{l} b_{l+1} \cdots b_{n-r}$, with $b_{l} \leq-2$. Since (3a) holds, by Proposition 18, the thesis follows.

Proposition 20. Consider ((1.1a) AND (2C) AND (3a)) $\Rightarrow$ absurd.

Proof. By Proposition 19, we have that $w_{-}^{\prime}=w_{-}$. Moreover, by (1.1a) in (30), we also have $w_{+}^{\prime}=w_{+}$because $\left\|w^{\prime}\right\|=\|w\|=$ $d$ and $w_{-}^{\prime}=w_{-}$; therefore, $w=w^{\prime}$. This is absurd because $w^{\prime}>w$ by hypothesis. 
Proposition 21. Consider ((1.1b) AND (2C) AND (3a)) $\Rightarrow$ absurd.

Proof. By (1.1b) in (12), we know that $w=0 \cdots 0 \mid b_{1} \cdots b_{n-r}$, and this implies $\left\|w_{+}\right\|=0$ and $\left\|w_{-}\right\|=d$. Moreover, by Proposition 19, $w_{-}^{\prime}=w_{-}$; therefore it follows that $\left\|w_{-}^{\prime}\right\|=d$; hence $\left\|w_{+}^{\prime}\right\|=0$ since $w^{\prime} \in S(n, d, r)$. This shows that $w=w^{\prime}$ and this is absurd because $w>w^{\prime}$.

Proposition 22. Consider ((1.1a) AND (2B)) $\Rightarrow w_{+}^{\prime}=w_{+}$.

Proof. By (1.1a) in (30) and (2B), it follows that $w=a_{r}$. $a_{l+1} 10 \cdots 0 \mid b_{1} \cdots b_{n-r}$ and $w^{\prime}=a_{r} \cdots a_{l+1} 1 a_{l-1}^{\prime} \cdots a_{1}^{\prime} \mid b_{1} \cdots b_{n-r}$. Now, since $w^{\prime} \in S(n, r)$ and $a_{l}=a_{l}^{\prime}=1$, we have that $1=a_{l}^{\prime}>$ $a_{l-1}^{\prime} \geq \cdots \geq a_{1}^{\prime} \geq 0$; that is, $a_{l-1}^{\prime}=\cdots=a_{1}^{\prime}=0$. Therefore $w_{+}^{\prime}=w_{+}$.

Proposition 23. Consider ((1.1a) AND (2B) AND (3.1b)) $\Rightarrow$ absurd.

Proof. By Proposition $22 w_{+}^{\prime}=w_{+}$and by Proposition $8 w=$ $w_{-}^{\prime}$; hence $w=w^{\prime}$ and this is a contradiction.

Proposition 24. Consider ((1.1a) AND (3.1b)) $\Rightarrow$ absurd.

Proof. By Proposition $8, w=w_{-}^{\prime}=\mid 00 \cdots 0$; hence $\left\|w_{+}^{\prime}\right\|=$ $\left\|w_{+}\right\|=d$ because $w, w^{\prime} \in S(n, d, r)$. Moreover, by (1.1a) in (30), it follows that

$$
w_{+}^{\prime}=w_{+}=a_{r} \cdots a_{l+1} a_{l} 0 \cdots 0 .
$$

Therefore, $w=w^{\prime}$, which is absurd.

Proposition 25. Consider ((1.1a) AND (2B) AND (3a)) $\Rightarrow$ absurd.

Proof. By Proposition 18, we have $w=a_{r} \cdots a_{1} \mid 0 \cdots 0 b_{l} b_{l+1} \cdots$ $b_{n-r}$ with $b_{l}<0$ and $l \geq 2$. By Proposition 22, $w_{+}^{\prime}=w_{+}$. Now, if $b_{l} \leq-2$, by Proposition 18 we have $w_{-}^{\prime}=w_{-}$and therefore $w^{\prime}=w$, which is absurd. So we can assume that $b_{l}=-1$. Since $w_{+}^{\prime}=w_{+},\left\|w^{\prime}\right\|=\|w\|=d$ and $w \sqsubset w^{\prime}$, it follows that $b_{l}^{\prime}$ cannot be 0 ; otherwise, it is $\left\|w^{\prime}\right\|<d$. This implies that it must be $b_{l}^{\prime}=-1$. We distinguish now two cases.

Case 1. If $l \in\{2, \ldots, n-r-1\}$, since $b_{l}=b_{l}^{\prime}=-1$, by Lemma 17 (with $q=l$ ) we obtain $b_{l+1}=b_{l+1}^{\prime}<0$. Now, if $l+1=n-r$, then $w_{-}=w_{-}^{\prime}$, and as before this provides a contradiction. If $l+1<n-r$, we can still apply Lemma 17 (with $q=l+1$ ) and therefore have $b_{l+2}=b_{l+2}^{\prime}<0$. Proceeding in this way in all cases we always obtain $w_{-}=w_{-}^{\prime}$ and hence is absurd.

Case 2. If $l=n-r$, then $w=a_{r} \cdots a_{1} \mid 0 \cdots 0(-1)$, and since $\left\|w^{\prime}\right\|=\|w\|=d, w\left[w^{\prime}\right.$, and $w_{+}^{\prime}=w_{+}$, it must also be $w^{\prime}=a_{r} \cdots a_{1} \mid 0 \cdots 0(-1)$; that is, $w=w^{\prime}$, absurd.

Proposition 26. Consider ((1.1a) AND (2B) AND (3.2b)) $\Rightarrow$ absurd.
Proof. By Proposition 22, $w_{+}^{\prime}=w_{+}$. Then by (3.2b) in (16), since $w_{+}^{\prime}=w_{+},\left\|w_{-}^{\prime}\right\|=\left\|w_{-}\right\|$, and $w \sqsubset w^{\prime}$, it must also be

$$
w_{-}^{\prime}=\mid 00 \cdots 0(-1)(-2) \cdots(-l)
$$

Hence we obtain $w=w^{\prime}$, absurd.

Proposition 27. Consider ((1.2b) AND (2C) AND (3a)) $\Rightarrow$ absurd.

Proof. By Proposition 19, $w_{-}^{\prime}=w_{-}$. Then, since $w \sqsubset w^{\prime}$, $\left\|w^{\prime}\right\|=\|w\|$, and $w_{-}^{\prime}=w_{-}$, by (1.2b) in (13) we deduce that $w_{+}^{\prime}=w_{+}$. Hence the absurd $w^{\prime}=w$.

Proposition 28. Consider ((2.1d) AND (3a)) $\Rightarrow$ absurd.

Proof. Since $w \subset w^{\prime}$ by (2.1d) in (14), it follows that $w_{+}^{\prime}=w_{+}$. Then, since (3a) in (29) holds, the same proof of Proposition 25 leads to the absurd $w=w^{\prime}$.

We complete now the proof of our main result; that is, we provide three syntactic trees where each possible path from the roots to the leaves leads to a contradiction. In the following we draw each tree and next we also write all the implication chains for each path from the roots to the leafs.

We recall at first some useful implications and equivalences already proved in the previous section:

$(\Theta 1 \mathrm{~A})\left(1 \mathrm{~A}^{\prime}\right) \Leftrightarrow \quad[$ by Proposition 13 (i)] $(1 \mathrm{~A}) \Rightarrow$ by Proposition 14] (1a) $\Rightarrow$ [by Proposition 16] (1.1a)

$(\Theta 2 \mathrm{~A})\left(2 \mathrm{~A}^{\prime}\right) \Rightarrow[$ by Proposition 12$]$ absurd

$(\Theta 2 \mathrm{D})(2 \mathrm{D}) \Leftrightarrow$ [by Proposition 6] (2.1d)

$(\Theta 3 A)\left(3 A^{\prime}\right) \Leftrightarrow[$ by Proposition 13 (ii) $](3 \mathrm{~A}) \Rightarrow$ by Proposition 14] (3a) $\Rightarrow$ [see statement of Proposition 18] Proposition 18.

The first tree that we consider is that having the condition $\left(1 \mathrm{~A}^{\prime}\right)$ as root (we denote such tree with TREE $\left(1 \mathrm{~A}^{\prime}\right)$ ). TREE $\left(1 \mathrm{~A}^{\prime}\right)$ :

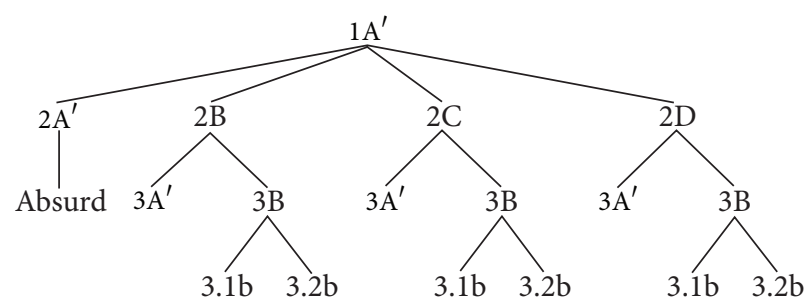

We observe now that by virtue of Proposition 5 when we start from the condition (1B), we must consider two subtrees, the first with root (1.1b) (which we denote by TREE(1.1b)) that is 


\section{TREE (1.1b):}

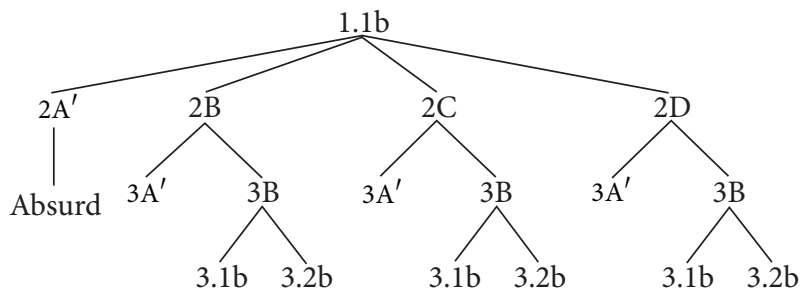

and the second (which we denote by TREE(1.2b)) that is TREE (1.2b):

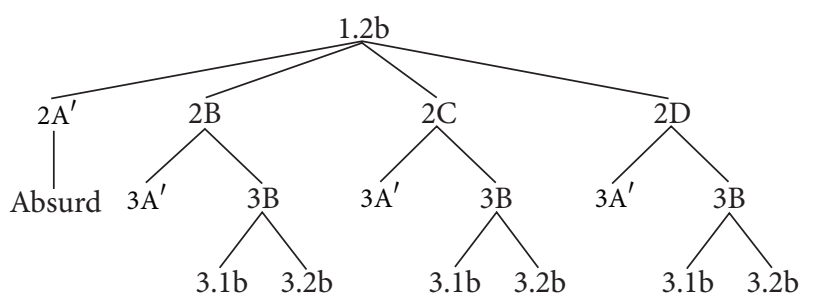

Let us note that the ramification

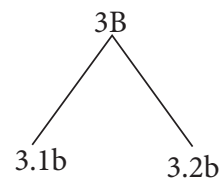

in each of the previous trees follows by Proposition 7 .

We have then exactly 27 possible paths from the roots to the leafs in all the previous trees (we exclude the paths that lead to $\left(2 \mathrm{~A}^{\prime}\right)$ because we already know that they lead to a contradiction by $\mathrm{\Theta} 2 \mathrm{~A})$. In the following we write all these 27 paths and, for each, the related proposition that leads to a contradiction together with some implication among the $\Theta 1 \mathrm{~A}, \Theta 2 \mathrm{D}$, and $\Theta 3 \mathrm{~A}$. In the sequel, the node (3B) is omitted for brevity because it is only an intermediate node and in the paths that involve (3B) we use only the leafs (3.1b) and (3.2b) to deduce the related contradictions:

$\left(1 \mathrm{~A}^{\prime}\right)$ AND $(2 \mathrm{~B})$ AND $\left(3 \mathrm{~A}^{\prime}\right) \Rightarrow$ absurd by $\Theta 1 \mathrm{~A}, \Theta 3 \mathrm{~A}$, and Proposition 25

$\left(1 \mathrm{~A}^{\prime}\right)$ AND $(2 \mathrm{~B})$ AND $(3.1 \mathrm{~b}) \Rightarrow$ absurd by $\Theta 1 \mathrm{~A}$ and Proposition 23

$\left(1 \mathrm{~A}^{\prime}\right)$ AND (2B) AND (3.2b) $\Rightarrow$ absurd by $\Theta 1 \mathrm{~A}$ and Proposition 26

$\left(1 \mathrm{~A}^{\prime}\right)$ AND $(2 \mathrm{C})$ AND $\left(3 \mathrm{~A}^{\prime}\right) \Rightarrow$ absurd by $\Theta 1 \mathrm{~A}, \Theta 3 \mathrm{~A}$, and Proposition 20

$\left(1 \mathrm{~A}^{\prime}\right)$ AND (2C) AND (3.1b) $\Rightarrow$ absurd by $\Theta 1 \mathrm{~A}$ and Proposition 24

$\left(1 \mathrm{~A}^{\prime}\right)$ AND (2C) AND (3.2b) $\Rightarrow$ absurd by $\Theta 1 \mathrm{~A}$ and Proposition 9 (iii)

$\left(1 \mathrm{~A}^{\prime}\right)$ AND (2D) AND $\left(3 \mathrm{~A}^{\prime}\right) \Rightarrow$ absurd by $\Theta 2 \mathrm{D}, \Theta 3 \mathrm{~A}$, and Proposition 28 $\left(1 \mathrm{~A}^{\prime}\right)$ AND (2D) AND (3.1b) $\Rightarrow$ absurd by $\Theta 1 \mathrm{~A}$ and Proposition 24

$\left(1 \mathrm{~A}^{\prime}\right)$ AND (2D) AND (3.2b) $\Rightarrow$ absurd by $\Theta 2 \mathrm{D}$ and Proposition 11

(1.1b) AND (2B) AND (3A') $\Rightarrow$ absurd by Proposition 9 (i)

(1.1b) AND (2B) AND (3.1b) $\Rightarrow$ absurd by Proposition 9 (i)

(1.1b) AND (2B) AND (3.2b) $\Rightarrow$ absurd by Proposition 9 (i)

(1.1b) AND (2C) AND $\left(3 \mathrm{~A}^{\prime}\right) \Rightarrow$ absurd by $\Theta 3 \mathrm{~A}$ and by Proposition 21

(1.1b) AND (2C) AND (3.1b) $\Rightarrow$ absurd by Proposition 9 (iv)

(1.1b) AND (2C) AND (3.2b) $\Rightarrow$ absurd by Proposition 9 (iii)

(1.1b) AND (2D) AND (3A') $\Rightarrow$ absurd by $\Theta 2 \mathrm{D}$ and by Proposition 9 (ii)

(1.1b) AND (2D) AND (3.1b) $\Rightarrow$ absurd by $\Theta 2 \mathrm{D}$ and by Proposition 9 (ii)

(1.1b) AND (2D) AND (3.2b) $\Rightarrow$ absurd by $\Theta 2$ D and by Proposition 9 (ii)

(1.2b) AND (2B) AND $\left(3 \mathrm{~A}^{\prime}\right) \Rightarrow$ (1.2b) AND (2D) AND $\left(3 A^{\prime}\right)$ (that we examine below) by $\Theta 2 D$ and by Proposition 10

(1.2b) AND (2B) AND (3.1b) $\Rightarrow$ (1.2b) AND (2D) AND (3.1b) (that we examine below) by $\Theta 2 \mathrm{D}$ and by Proposition 10

(1.2b) AND (2B) AND (3.2b) $\Rightarrow$ (1.2b) AND (2D) AND (3.2b) (that we examine below) by $\Theta 2 \mathrm{D}$ and by Proposition 10

(1.2b) AND (2C) AND (3A') $\Rightarrow$ absurd by $\Theta 3$ A and by Proposition 27

(1.2b) AND (2C) AND (3.1b) $\Rightarrow$ absurd by Proposition 9 (v)

(1.2b) AND (2C) AND (3.2b) $\Rightarrow$ absurd by Proposition 9 (iii)

(1.2b) AND (2D) AND $\left(3 \mathrm{~A}^{\prime}\right) \Rightarrow$ absurd by $\Theta 2 \mathrm{D}, \Theta 3 \mathrm{~A}$, and Proposition 28

(1.2b) AND (2D) AND (3.1b) $\Rightarrow$ absurd by Proposition 9 (v)

(1.2b) AND (2D) AND (3.2b) $\Rightarrow$ absurd by $\Theta 2$ D and by Proposition 11

This concludes the proof of Theorem 4. Below we draw the Hasse diagram of the lattice $S(5,3,2)$ by using the evolution Rules 1, 2, and 3 starting with the minimum element of this lattice, which is $00 \mid 123$. We label a generic edge of the 
next diagram with the symbol $R k$ if it leads to a production that uses the Rule $k$, for $k \in\{1,2,3\}$ :

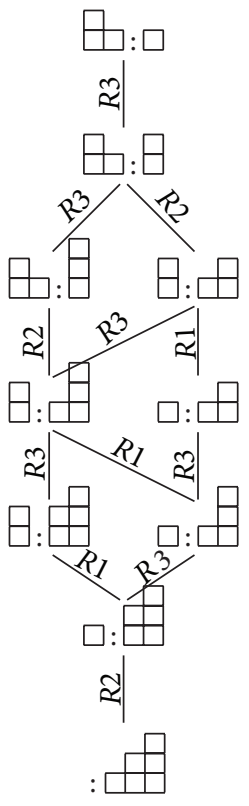

\section{References}

[1] D. K. Arrowsmith and C. M. Place, An Introduction to Dynamical Systems, Cambridge University Press, Cambridge, UK, 1990.

[2] J. L. G. Guirao, F. L. Pelayo, and J. C. Valverde, "Modeling the dynamics of concurrent computing systems," Computers \& Mathematics with Applications, vol. 61, no. 5, pp. 1402-1406, 2011.

[3] W. Foryś, J. L. G. Guirao, and P. Oprocha, "A dynamical model of parallel computation on bi-infinite time-scale," Journal of Computational and Applied Mathematics, vol. 235, no. 7, pp. 1826-1832, 2011.

[4] J. A. Aledo, S. Martínez, and J. C. Valverde, "Parallel dynamical systems over directed dependency graphs," Applied Mathematics and Computation, vol. 219, no. 3, pp. 1114-1119, 2012.

[5] J. A. Aledo, S. Martinez, and J. C. Valverde, "Parallel discrete dynamical systems on independent local functions," Journal of Computational and Applied Mathematics, vol. 237, no. 1, pp. 335339, 2013.

[6] J. A. Aledo, S. Martínez, F. L. Pelayo, and J. C. Valverde, "Parallel discrete dynamical systems on maxterm and minterm Boolean functions," Mathematical and Computer Modelling, vol. 55, no. 3-4, pp. 666-671, 2012.

[7] C. Bisi and G. Chiaselotti, "A class of lattices and boolean functions related to the Manickam-Miklös-Singhi conjecture," Advances in Geometry, vol. 13, no. 1, pp. 1-27, 2013.

[8] G. E. Andrews, "Euler's 'De Partitio numerorum,' Bulletin of American Mathematical Society, vol. 44, no. 4, pp. 561-573, 2007.

[9] W. J. Keith, "A bijective toolkit for signed partitions," Annals of Combinatorics, vol. 15, no. 1, pp. 95-117, 2011.

[10] G. Chiaselotti, G. Marino, and C. Nardi, "A minimum problem for finite sets of real numbers with nonnegative sum," Journal of Applied Mathematics, vol. 2012, Article ID 847958, 15 pages, 2012.

[11] T. Brylawski, “The lattice of integer partitions," Discrete Mathematics, vol. 6, pp. 201-219, 1973.

[12] K. J. Al-Agha and R. J. Greechie, “The involutory dimension of involution posets," Order, vol. 18, no. 4, pp. 323-337, 2002.

[13] K. Brenneman, R. Haas, and A. G. Helminck, "Implementing an algorithm for the twisted involution poset for Weyl groups," in Proceedings of the 37th Southeastern International Conference on Combinatorics, Graph Theory and Computing, vol. 182, pp. 137-144, 2006. 


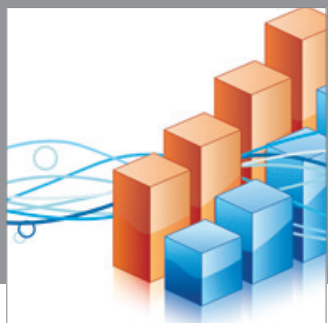

Advances in

Operations Research

mansans

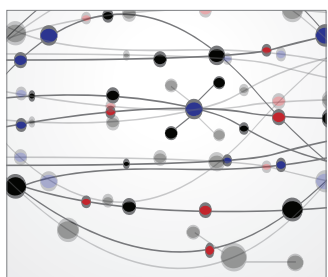

The Scientific World Journal
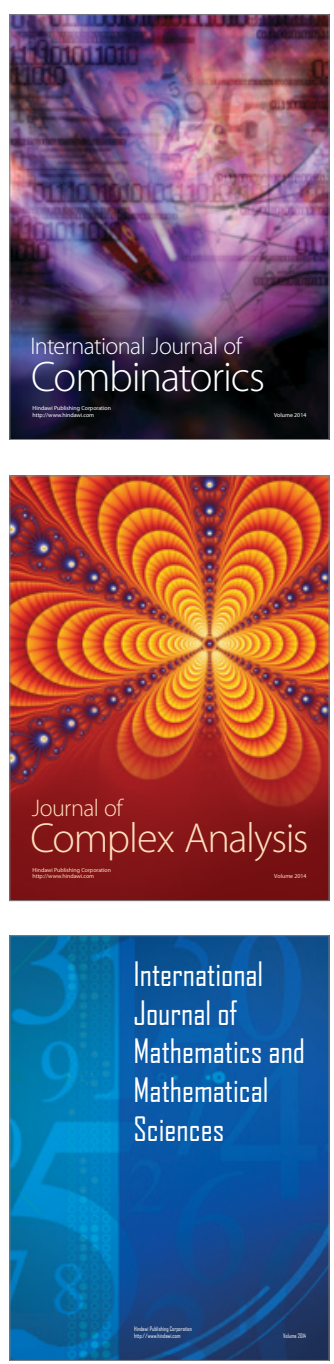
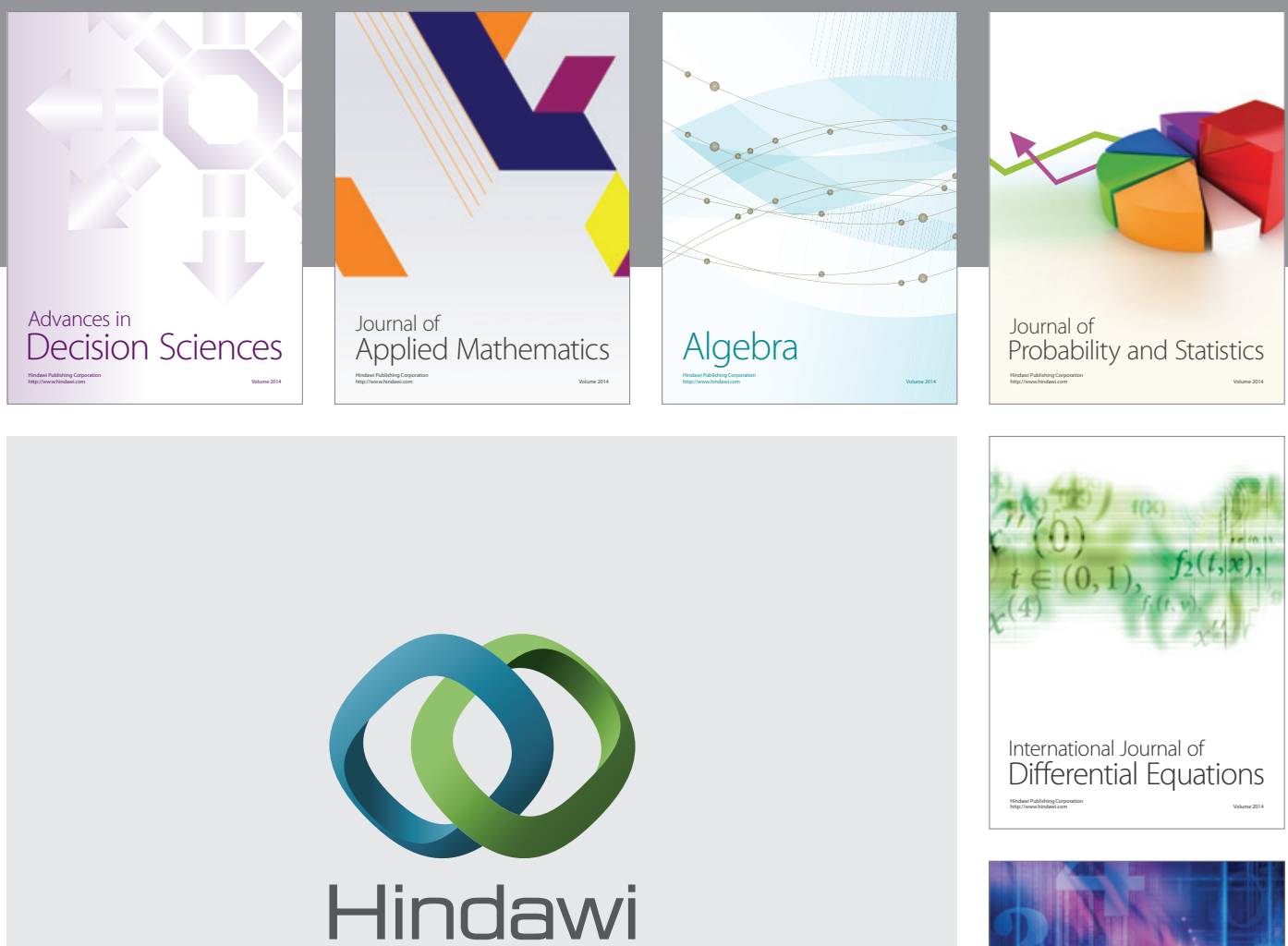

Submit your manuscripts at http://www.hindawi.com
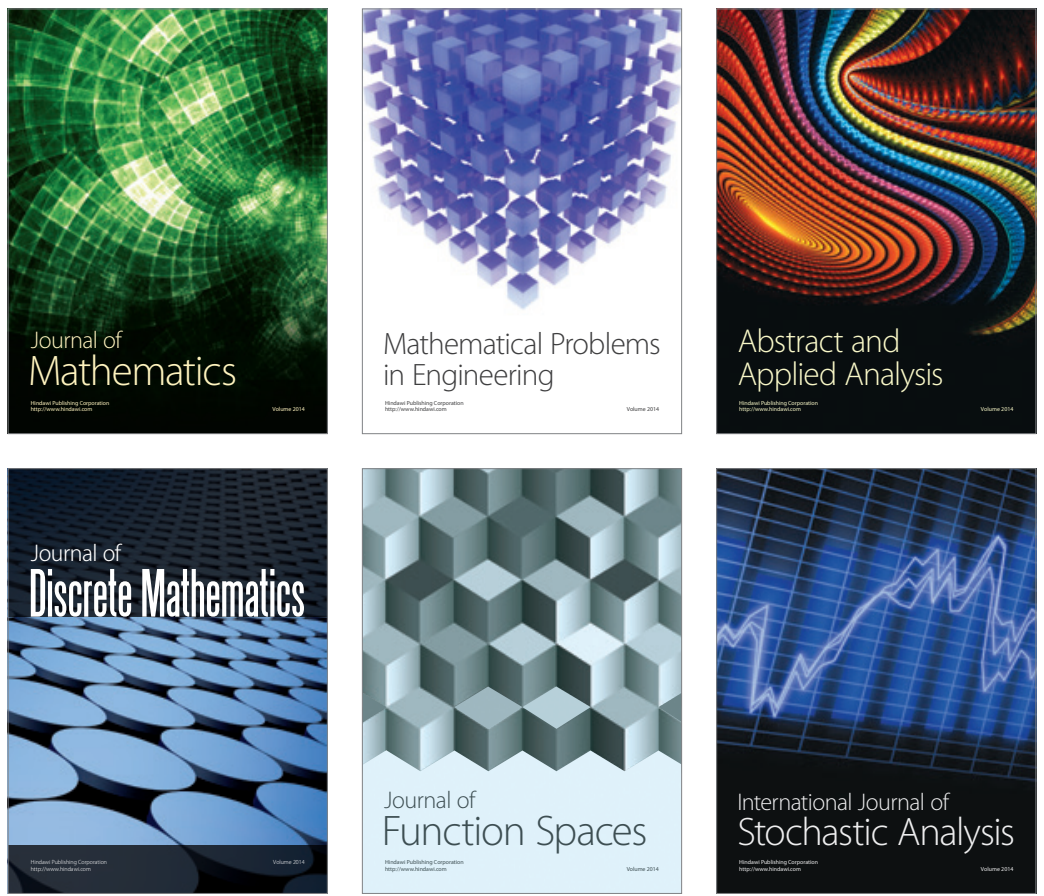

Journal of

Function Spaces

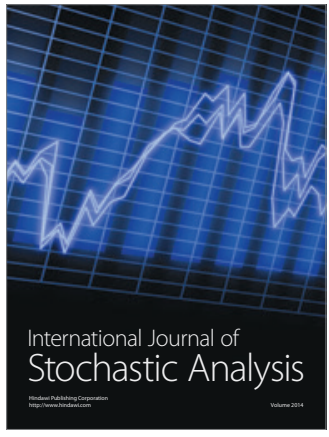

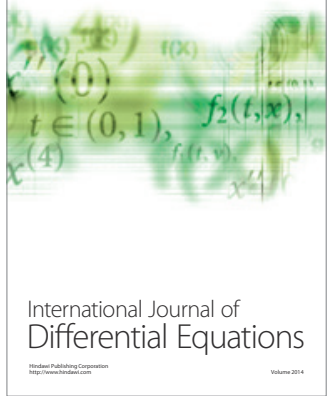
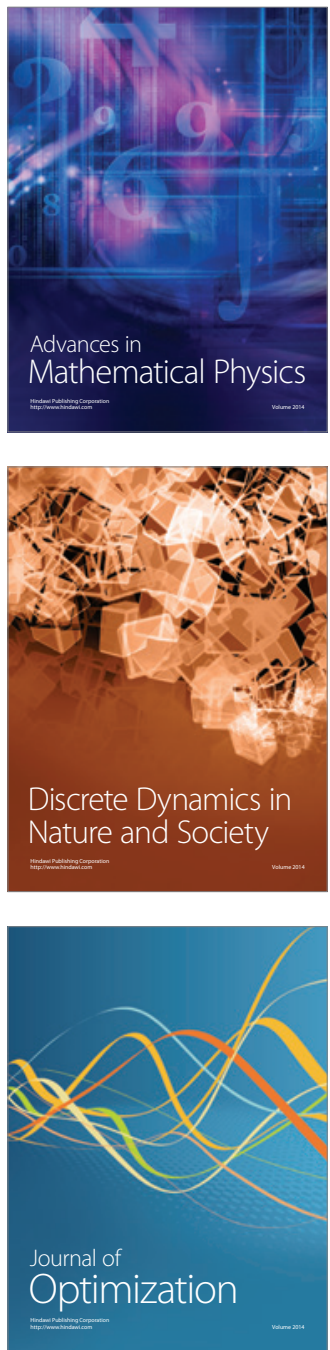